\title{
A POSTERIORI ERROR ESTIMATES OF FINITE VOLUME ELEMENT METHOD FOR SECOND-ORDER QUASILINEAR ELLIPTIC PROBLEMS
}

\author{
CHUNJIA BI AND CHENG WANG*
}

\begin{abstract}
In this paper, we consider the a posteriori error estimates of the finite volume element method for the general second-order quasilinear elliptic problems over a convex polygonal domain in the plane, propose a residual-based error estimator and derive the global upper and local lower bounds on the approximation error in the $H^{1}$-norm. Moreover, for some special quasilinear elliptic problems, we propose a residual-based a posteriori error estimator and derive the global upper bound on the error in the $L^{2}$-norm. Numerical experiments are also provided to verify our theoretical results.
\end{abstract}

Key words. quasilinear elliptic problem, finite volume element method, a posteriori error estimates.

\section{Introduction}

The finite volume element method (FVEM, also called finite volume method or covolume method in some literature) is a class of important numerical tools for solving differential equations, especially for those arising from physical conservation laws including mass, momentum, and energy. Because this method possesses local physical conservation property, which is crucial in many applications, it is popular in computational fluid mechanics. In the past several decades, many researchers have studied this method extensively and obtained some important results. We refer to monograph [30] for the general presentation of this method, and to [3, 10, $11,12,13,14,15,16,17,18,22,23,26,27,28,29,31,32,35,36,38,39,40,41,42]$ and references therein for details.

In this paper, we study the a posteriori error estimates of the finite volume element method for the second-order quasilinear elliptic boundary value problems

$$
\left\{\begin{array}{r}
\mathcal{L} u=-\nabla \cdot F(x, \nabla u)+g(x, u, \nabla u)=0, \quad \text { in } \quad \Omega, \\
u=0, \quad \text { on } \partial \Omega,
\end{array}\right.
$$

where $\Omega$ is a convex polygonal domain in $\mathbb{R}^{2}$ with the boundary $\partial \Omega$. We assume that $F(x, z): \bar{\Omega} \times \mathbb{R}^{2} \rightarrow \mathbb{R}^{2}$ and $g(x, y, z): \bar{\Omega} \times \mathbb{R}^{1} \times \mathbb{R}^{2} \rightarrow \mathbb{R}^{1}$ are smooth functions and that (1) has a solution $u \in H_{0}^{1}(\Omega) \cap W^{2, r}(\Omega)$ for some $r>2$. The smoothness requirements on those functions will be given in detail later.

There are some important numerical results available for (1). We refer the reader to $[20,33,37]$ for the finite element method and to [25] for the $h p$-discontinuous Galerkin methods.

Received by the editors August 30, 2015.

2000 Mathematics Subject Classification. 65N15; 65N30.

* Corresponding author.

C. Bi is partially supported by National Science Foundation of China (11571297) and Shandong Province Natural Science Foundation (ZR2014AM003). C. Wang is partially supported by National Science Foundation of China (11101311). 
Until now, the finite volume element method for the linear elliptic problems has been well understood. However, there are very few works related to the nonlinear elliptic problems. To the best of our knowledge, the authors of $[5,6,14,29]$ studied the finite volume element method and developed some a priori error estimates only for the following quasilinear elliptic problems

$$
-\nabla \cdot(\lambda(u) \nabla u)=f(x), \quad x \in \Omega, \quad u(x)=0, \quad x \in \partial \Omega,
$$

where $\lambda$ is a smooth scalar function. Recently, Bi and Ginting [8] considered the finite volume element method for (1), proved the existence and uniqueness of the finite volume element solutions under the assumption $u \in W^{2, r}(\Omega), r>2$, where $r$ may be close to 2 , and derived the a priori error estimates in the $H^{1}-, L^{2}-, W^{1, \infty_{-}}$ and $L^{\infty}$-norms.

Compared with the relatively mature a posteriori error estimates of the finite element method, the a posteriori error analysis of the finite volume element method is still under development, and until now only a few results have been obtained. We mention $[12,28]$ for the linear elliptic problems. However, for the nonlinear elliptic problems, there are only [4] and [7] available. The authors of [4, 7] established the residual-based a posteriori error estimates of the finite volume element method, respectively, for (2) and

$$
-\nabla \cdot(A(u) \nabla u)=f(x), \quad x \in \Omega, \quad u(x)=0, \quad x \in \partial \Omega,
$$

where $A(u)$ is a smooth and bounded uniformly positive definite matrix.

As a subsequent work of [8], in this paper, we study the a posteriori error estimates of the finite volume element method for (1) and propose a natural and computationally easy residual-based $H^{1}$-norm a posteriori error estimator. Under two assumptions that $u \in W^{2, r}(\Omega), r>2$, and the mesh parameter is sufficiently small, we derive the global upper bound and local lower bounds on the error. Moreover, for some special problems (1) which satisfy $D_{z z} F=0$ and $D_{z z} g=0$, we propose a residual-based $L^{2}$-norm a posteriori error estimator and derive the global upper bound on the error. We point out that the two assumptions above are reasonable, which guarantee the existence of the finite volume element approximations of (1), see [8] for details.

In the present work, for the sake of simplicity, we focus our attention on the quasilinear problems on a polygonal domain, which is the same as those in [20, $33,37]$. Smooth boundaries are important for many nonlinear problems as even theoretical results are not always available on polygonal domains. However, the proper treatment of the curved boundary is somewhat technical (see [21] for details) and we don't wish to clutter our presentation.

The organization of this paper is as follows. In section 2, we introduce some notation, formulate the finite volume element method for (1), and give some lemmas used in the subsequent analysis. In section 3 , we propose a residual-based $H^{1}$-norm a posteriori error estimator of the finite volume element method for (1) and derive the global upper bound and local lower bounds on the error. In section 4, for some special problems (1) which satisfy $D_{z z} F=0$ and $D_{z z} g=0$, such as Bratu's equation and some nonlinear eigenvalue equations, we propose a residual-based $L^{2}$ norm a posteriori error estimator and derive the global upper bound on the error. In section 5, we provide two numerical experiments that confirm our theoretical findings in this paper. Finally, in Section 6, we summarize the main results of this paper and draw some conclusions. 
Throughout this paper, $C$, with or without subscripts, denotes a generic positive constant independent of the mesh parameter and may be dependent on the exact solution of (1) and different at its different occurrences.

\section{Preliminaries}

2.1. Quasi-linear elliptic problems. For integer $m \geq 0$ and real number $1 \leq$ $p \leq \infty$, we employ the standard notation for the Sobolev spaces $W^{m, p}(\Omega)$, with the norm $\|\left.\cdot\right|_{m, p, \Omega}$ and the seminorm $|\cdot|_{m, p, \Omega}[9,19]$. In order to simplify the notation, we denote $W^{m, 2}(\Omega)$ by $H^{m}(\Omega)$ and skip the index $p=2$ and $\Omega$ whenever possible, i.e., we will use $\|u\|_{m, p, \Omega}=\|u\|_{m, p},\|u\|_{m, 2, \Omega}=\|u\|_{m}$ and $\|u\|_{0}=\|u\|$. The same convention is used for the seminorms as well. In addition, the space $H_{0}^{1}(\Omega)$ is defined, as usual, by $H_{0}^{1}(\Omega)=\left\{v \in H^{1}(\Omega) \mid v=0\right.$ on $\left.\partial \Omega\right\}$. In what follows, $(\cdot, \cdot)$ denotes the $L^{2}(\Omega)$ inner product.

We assume that the functions $F_{i}(x, z), i=1,2$ and $g(x, y, z)$ are twice continuously differentiable with all the derivatives through second order being bounded, i.e., there exists a constant $\beta>0$ such that

$$
\left|D F_{i}\right|+\left|D^{2} F_{i}\right|+|D g|+\left|D^{2} g\right| \leq \beta, \quad i=1,2 .
$$

Further, for any $\omega \in W^{1, \infty}(\Omega)$, we denote

$$
\begin{gathered}
a(\omega)=D_{z} F(x, \nabla \omega) \in \mathbb{R}^{2 \times 2}, \\
c(\omega)=D_{z} g(x, \omega, \nabla \omega) \in \mathbb{R}^{2}, \quad d(\omega)=D_{y} g(x, \omega, \nabla \omega) \in \mathbb{R}^{1} .
\end{gathered}
$$

The linearized operator $\mathcal{L}^{\prime}$ at $\omega$ (namely the Fréchet derivative of $\mathcal{L}$ at $\omega$ ) is then given by

$$
\mathcal{L}^{\prime}(\omega) v=-\nabla \cdot(a(\omega) \nabla v)+c(\omega) \cdot \nabla v+d(\omega) v
$$

As in [37], the basic assumptions are, first of all, for the solution $u$ of (1),

$$
\alpha_{0}|\xi|^{2} \leq \xi^{T} a(u) \xi, \quad \forall \xi \in \mathbb{R}^{2}, x \in \bar{\Omega}
$$

for some constant $\alpha_{0}>0$, and secondly $\mathcal{L}^{\prime}(u): H_{0}^{1}(\Omega) \rightarrow H^{-1}(\Omega)$ is an isomorphism. As a result of these assumptions, $u$ must be an isolated solution of (1). A simple sufficient condition for the assumption that $\mathcal{L}^{\prime}(u)$ is an isomorphism is $d(u) \geq 0$, see [37] for details.

Problems (1) arise in several areas of applications. Keeping in mind the assumption $u \in W^{2, r}(\Omega), r>2$, from the Sobolev embedding inequality $\|u\|_{1, \infty} \leq$ $C\|u\|_{2, r} \leq C_{1}$, we know that the following quasi-linear elliptic problems can be treated with the technique presented in this paper.

1. The equation of prescribed mean curvature:

$$
F(x, \nabla u)=\left(1+|\nabla u|^{2}\right)^{-\frac{1}{2}} \nabla u, \quad g(x, u, \nabla u)=g(x) .
$$

2. The subsonic flow of an irrotational, ideal, compressible gas:

$$
F(x, \nabla u)=\left[1-\frac{\gamma-1}{2}|\nabla u|^{2}\right]^{1 /(\gamma-1)} \nabla u, \quad g(x, u, \nabla u)=g(x), \quad \gamma>1 .
$$

3. Bratu's equation:

$$
F(x, \nabla u)=\nabla u, \quad g(x, u, \nabla u)=\lambda e^{u}, \quad \lambda>0 .
$$

4. A nonlinear eigenvalue problem:

$$
F(x, \nabla u)=\nabla u, \quad g(x, u, \nabla u)=\lambda u-u^{\kappa}, \quad \lambda>0, \quad \kappa \geq 2 .
$$


2.2. Finite volume element method. For the polygonal domain $\Omega$, we consider a conforming triangulation $\mathcal{T}_{h}$ consisting of closed triangle element $K$ such that $\bar{\Omega}=\cup_{K \in \mathcal{T}_{h}} K$, where $h=\max _{K \in \mathcal{T}_{h}}\left\{h_{K}\right\}$ and $h_{K}$ is the diameter of the triangle $K$. We assume that $\mathcal{T}_{h}$ is shape regular $[9,19]$, that is, there exists a positive constant $C$ such that $\left|h_{K}\right| \leq C \rho_{K}, \forall K \in \mathcal{T}_{h}$, where $\rho_{K}$ is the diameter of the largest ball contained in $K$. We denote by $\mathcal{E}_{h}^{0}, N_{h}$ and $N_{h}^{0}$ the sets of all interior edges, all vertices and all interior vertices of $\mathcal{T}_{h}$, respectively.

In order to formulate the finite volume element method for solving (1), we first introduce a dual mesh $\mathcal{T}_{h}^{*}$ based on $\mathcal{T}_{h}$ whose elements are called the control volumes. In this paper, we construct the control volume in the same way as in $[5,6,14,22,26,30]$. Let $Q_{K}$ be the barycenter of an element $K \in \mathcal{T}_{h}$. We connect $Q_{K}$ with line segments to the midpoints of the edges of $K$, thus partitioning $K$ into three quadrilaterals $K_{p}, q \in N_{h}(K)$, where $N_{h}(K)$ is the set of the vertices of $K$. Then with each vertex $p \in N_{h}$ we associate a control volume $K_{p}^{*}$, which consists of the union of the subregions $K_{p}$, sharing the vertex $p$. Finally, we obtain a group of control volumes covering the domain $\Omega$, which is called the dual mesh $\mathcal{T}_{h}^{*}$ of $\mathcal{T}_{h}$. We call the control volume mesh $\mathcal{T}_{h}^{*}$ regular or quasi-uniform, if there exists a positive constant $C>0$ such that

$$
C^{-1} h^{2} \leq \operatorname{meas}\left(K_{p}^{*}\right) \leq C h^{2}, \quad \forall K_{p}^{*} \in \mathcal{T}_{h}^{*} .
$$

The barycenter-type dual partition can be introduced for any triangulation $\mathcal{T}_{h}$ and leads to relatively simple calculations. In addition, if the triangulation $\mathcal{T}_{h}$ is quasiuniform, then the dual partition $\mathcal{T}_{h}^{*}$ is also quasi-uniform.

We define the standard linear finite element space $S_{h}$ as

$$
S_{h}=\left\{v_{h} \in C(\bar{\Omega}):\left.v_{h}\right|_{K} \text { is linear for all } K \in \mathcal{T}_{h} \text { and }\left.v_{h}\right|_{\partial \Omega}=0\right\},
$$

and its dual volume element space $S_{h}^{*}$ by

$$
S_{h}^{*}=\left\{v_{h} \in L^{2}(\Omega):\left.v_{h}\right|_{K_{p}^{*}} \text { is constant for all } K_{p}^{*} \in \mathcal{T}_{h}^{*} \text { and }\left.v_{h}\right|_{K_{p}^{*}}=0, \forall p \in \partial \Omega\right\} .
$$

Let $I_{h}^{*}: C(\Omega) \cap H_{0}^{1}(\Omega) \rightarrow S_{h}^{*}$ be the piecewise constant interpolation operator defined by

$$
I_{h}^{*} v_{h}=\sum_{p \in N_{h}^{0}} v_{h}(p) \chi_{K_{p}^{*}},
$$

where $\chi_{K_{p}^{*}}$ is the characteristic function of the control volume $K_{p}^{*}$.

The finite volume element solution of (1) is based upon the conservation property

$$
-\int_{\partial K_{p}^{*}} F(x, \nabla u) \cdot \mathbf{n} \mathrm{d} s+\int_{K_{p}^{*}} g(x, u, \nabla u) \mathrm{d} x=0,
$$

which is obtained from integrating (1) over the control volume $K_{p}^{*}$ and applying Green's formula. Here and elsewhere $\mathbf{n}$ is the outer-normal vector of the associated integration domain. The finite volume element method is to find $u_{h} \in S_{h}$ satisfying

$$
-\int_{\partial K_{p}^{*}} F\left(x, \nabla u_{h}\right) \cdot \mathbf{n} \mathrm{d} s+\int_{K_{p}^{*}} g\left(x, u_{h}, \nabla u_{h}\right) \mathrm{d} x=0, \quad \forall K_{p}^{*} \in \mathcal{T}_{h}^{*} .
$$

For the purpose of analysis, we express (8) in a variational formulation with the help of the interpolation operator $I_{h}^{*}$. This is done by multiplying (8) by $v_{h}(p)$ and sum over all $p \in N_{h}^{0}$. Now we have the formulation as to find $u_{h} \in S_{h}$ such that

$$
a_{h}\left(u_{h}, I_{h}^{*} v_{h}\right)=0, \quad \forall v_{h} \in S_{h},
$$


where for every $u_{h}, v_{h} \in S_{h}$,

$a_{h}\left(u_{h}, I_{h}^{*} v_{h}\right)=-\sum_{p \in N_{h}^{0}} v_{h}(p) \int_{\partial K_{p}^{*}} F\left(x, \nabla u_{h}\right) \cdot \mathbf{n} \mathrm{d} s+\sum_{p \in N_{h}^{0}} v_{h}(p) \int_{K_{p}^{*}} g\left(x, u_{h}, \nabla u_{h}\right) \mathrm{d} x$.

At this stage, we list several lemmas that are used in the subsequent analysis.

Lemma 2.1. ([13,35]) For $K \in \mathcal{T}_{h}$ and $E$ an edge of $K$, each $v_{h} \in S_{h}$ satisfies the following:

$$
\begin{gathered}
\int_{K}\left(v_{h}-I_{h}^{*} v_{h}\right) \mathrm{d} x=0 \quad \text { and } \quad \int_{E}\left(v_{h}-I_{h}^{*} v_{h}\right) \mathrm{d} s=0 \\
\| v_{h}-I_{h}^{*} v_{h}||_{0, q, K} \leq C h_{K}\left|v_{h}\right|_{1, q, K} \quad \text { and } \quad \| v_{h}-\left.I_{h}^{*} v_{h}\right|_{0, q, E} \leq C h_{K}^{1-\frac{1}{q}}\left|v_{h}\right|_{1, q, K},
\end{gathered}
$$

where $1 \leq q \leq \infty$.

The following lemma gives the existence and uniqueness of finite volume element solution of (9), which is proved in [8].

Lemma 2.2.([8]) For sufficiently small $h$, the finite volume element equation (9) has a solution $u_{h}$ satisfying

$$
\left\|u-u_{h}\right\|_{1, \infty} \leq C h^{1-\frac{2}{r}}|\ln h|\left(1+|u|_{2, r}\right) .
$$

Furthermore there exists a constant $\varrho>0$ such that $u_{h}$ is the only solution satisfying $\left\|u-u_{h}\right\|_{1, \infty} \leq \varrho$.

The following error estimate in the $H^{1}$-norm will be used in this paper.

Lemma 2.3. ([8]) Assume that $u \in W^{2, r}(\Omega), r>2$, and $u_{h} \in S_{h}$ are the solutions of (1) and (9), respectively, that satisfy (10). Then, for sufficiently small $h$, there exists a constant $C$ independent of $h$ such that

$$
\left\|u-u_{h}\right\|_{1} \leq C h|u|_{2} .
$$

Based on Lemma 2.3, we establish the stability of the finite volume element solution $u_{h}$ in the $W^{1, \infty}$-norm as follows.

Lemma 2.4. Let $u \in W^{2, r}(\Omega) \cap H_{0}^{1}(\Omega), r>2$, and $u_{h} \in S_{h}$ be the solution of (1) and (9), respectively. Then, there exists a constant $C$ independent of $h$ such that

$$
\left\|u_{h}\right\|_{1, \infty} \leq C|| u \|_{2, r}
$$

Proof. Using the triangle inequality, we write

$$
\left\|u_{h}\right\|_{1, \infty} \leq\left\|u_{h}-u^{I}\right\|_{1, \infty}+\left\|u^{I}\right\|_{1, \infty},
$$

where $u^{I} \in S_{h}$ is the interpolant of $u$. In view of the interpolation theory [19] and the Sobolev imbedding theorem [9], $\|u\|_{1, \infty} \leq C\|u\|_{2, r}$, we have

$$
\begin{aligned}
\left\|u^{I}\right\|_{1, \infty} & \leq\left\|u-u^{I}\right\|_{1, \infty}+\|u\|_{1, \infty} \\
& \leq C\|u\|_{2}+C\|u\|_{2, r} \leq C\|u\|_{2, r} .
\end{aligned}
$$

Using the inverse inequality $[9,19]$, Lemma 2.3 and the interpolation theory, we get

$$
\begin{aligned}
\left\|u_{h}-u^{I}\right\|_{1, \infty} & \leq C h^{-1}\left\|u_{h}-u^{I}\right\|_{1} \\
& \leq C h^{-1}\left(\left\|u_{h}-u\right\|_{1}+\left\|u-u^{I}\right\|_{1}\right) \\
& \leq C\|u\|_{2} .
\end{aligned}
$$

Combining (11), (12) with (13) gives the desired result.

The following error estimate in the $W^{1, \infty}$-norm has been proven in [8]. 
Lemma 2.5. ([8]) Assume that $u \in W^{2, r}(\Omega), r>2$, and $u_{h} \in S_{h}$ are the solutions of (1) and (9), respectively. Then, for sufficiently small $h$, there exists a constant $C$ independent of $h$ such that

$$
\left\|u-u_{h}\right\|_{1, \infty} \leq C h^{1-\frac{2}{r}}|\ln h||u|_{2, r} .
$$

The following trace theorem, proved in [1], will be used in the subsequent analysis.

Lemma 2.6.([1]) Let $E$ be an edge of $K$. For each $\omega \in H^{1}(K)$, there exists a constant $C$ independent of $h_{E}$ such that

$$
\|\omega\|_{0, E}^{2} \leq C\left(h_{E}^{-1}\|\omega\|_{0, K}^{2}+h_{E}\|\nabla \omega\|_{0, K}^{2}\right) .
$$

\section{3. $H^{1}$-norm a posteriori error estimator}

In this section, we present a residual-based $H^{1}$-norm a posteriori error estimator of the finite volume element method for (1) and obtain the computable upper bound and local lower bounds on the error.

3.1. Global upper bound. As an auxiliary tool, we first introduce the weak formulation of (1): Find $u \in H_{0}^{1}(\Omega)$ such that

$$
a(u, v)=0, \quad \forall v \in H_{0}^{1}(\Omega),
$$

where

$$
a(u, v)=(F(x, \nabla u), \nabla v)+(g(x, u, \nabla u), v) .
$$

As in [37], we define two parameters $\delta_{1}$ and $\delta_{2}$

$$
\delta_{1}= \begin{cases}0, & \text { if } F_{z z}(x, z) \equiv 0, g_{z z}(x, y, z) \equiv 0 \\ 1, & \text { otherwise }\end{cases}
$$

and

$$
\delta_{2}= \begin{cases}0, & \text { if } \delta_{1}=0, g_{y z}(x, y, z) \equiv 0 \\ 1, & \text { otherwise }\end{cases}
$$

For fixed $\omega$, we also introduce the following bilinear form (induced by $\mathcal{L}^{\prime}(\omega)$ )

$$
a^{\prime}(\omega ; v, \varphi)=(a(\omega) \nabla v, \nabla \varphi)+(c(\omega) \cdot \nabla v+d(\omega) v, \varphi) .
$$

With above notation, Xu proved the following Lemma 3.1 in [37], which will be used in our a posteriori error analysis.

Lemma 3.1. ([37]) For any $\omega, \omega_{h}, v \in H_{0}^{1}(\Omega)$,

$$
a\left(\omega_{h}, v\right)=a(\omega, v)+a^{\prime}\left(\omega, \omega_{h}-\omega, v\right)+R\left(\omega, \omega_{h}, v\right)
$$

where the remainder $R$, for a given constant $M>0$ and the functions $\omega$ and $\omega_{h}$ satisfying $\|\omega\|_{1, \infty}+\left\|\omega_{h}\right\|_{1, \infty} \leq M$, satisfies the estimate

$$
\left|R\left(\omega, \omega_{h}, v\right)\right| \leq C\left(\left\|\varepsilon_{h}\right\|_{0,4}^{2}+\delta_{1}\left\|\nabla \varepsilon_{h}\right\|_{0,4}^{2}+\delta_{2} \| \varepsilon_{h} \nabla \varepsilon_{h}||\right)\|\nabla v\|,
$$

where $\varepsilon_{h}=\omega-\omega_{h}$ and the constant $C$ depends on $M$.

Using the isomorphism of $\mathcal{L}^{\prime}(u)$, we have the following sup condition of the bilinear form $a^{\prime}(u ; \cdot, \cdot)$, see also [32, 37], whose proof can be found in [2].

Lemma 3.2. Assume that $u \in W^{2, r}(\Omega), r>2$, is the solution of (1). For each $\omega \in H_{0}^{1}(\Omega)$, we have

$$
\|\omega\|_{1} \leq C \sup _{v \in H_{0}^{1}(\Omega)} \frac{a^{\prime}(u ; \omega, v)}{\|v\|_{1}}
$$


Next, we introduce the jump of a vector-valued function across an interior edge, which will be used in the definition of the a posteriori error estimators. Let $E$ be an interior edge shared by elements $K^{+}$and $K^{-}$and $q$ be a vector-valued function, that is smooth inside each element $K^{ \pm} \cdot q^{ \pm}$denote the traces of $q$ on $E$ taken from within the interior of $K^{ \pm}$, respectively. Then, the jump of $q$ on $E$ is given by,

$$
[q]=q^{+} \cdot \mathbf{n}_{K^{+}}+q^{-} \cdot \mathbf{n}_{K^{-}},
$$

where $\mathbf{n}_{K^{ \pm}}$denote the unit outward normal vector of $\partial K^{ \pm}$, respectively.

The following lemma gives a representation of the error $u-u_{h}$, which plays a key role in the a posteriori error analysis.

Lemma 3.3. Assume that $u$ and $u_{h}$ are the solutions of (1) and (9), respectively. Then for the error $e_{h}=u-u_{h}$ and any $v \in H_{0}^{1}(\Omega)$, we have

$$
\begin{aligned}
a^{\prime}\left(u ; e_{h}, v\right)= & \sum_{K \in \mathcal{T}_{h}}\left(\nabla \cdot F\left(x, \nabla u_{h}\right)-g\left(x, u_{h}, \nabla u_{h}\right), v-I_{h}^{*} v^{I}\right)_{K} \\
& -\sum_{E \in \mathcal{E}_{h}^{0}}\left(\left[F\left(x, \nabla u_{h}\right)\right], v-I_{h}^{*} v^{I}\right)_{E}+R\left(u, u_{h}, v\right) \\
= & \Pi_{1}+\Pi_{2}+\Pi_{3},
\end{aligned}
$$

where $v^{I} \in S_{h}$ is the interpolant of $v$.

Proof. Setting $\omega=u, \omega_{h}=u_{h}$ in (18) and noting $u, u_{h} \in H_{0}^{1}(\Omega)$, we have

$$
a^{\prime}\left(u ; e_{h}, v\right)=a^{\prime}\left(u ; u-u_{h}, v\right)=\left(a(u, v)-a\left(u_{h}, v\right)\right)+R\left(u, u_{h}, v\right) .
$$

Since $v \in H_{0}^{1}(\Omega)$, from (14), we have $a(u, v)=0$. Then, from this identity and (15), we have

$$
\begin{aligned}
a(u, v)-a\left(u_{h}, v\right)= & -a\left(u_{h}, v\right) \\
= & -a\left(u_{h}, v-v^{I}\right)-a\left(u_{h}, v^{I}\right) \\
= & \left(-\left(F\left(x, \nabla u_{h}\right), \nabla\left(v-v^{I}\right)\right)-\left(g\left(x, u_{h}, \nabla u_{h}\right), v-v^{I}\right)\right) \\
& -a\left(u_{h}, v^{I}\right) \\
= & \Sigma_{1}+\Sigma_{2} .
\end{aligned}
$$

By Green's formula,

$$
\begin{aligned}
-\left(F\left(x, \nabla u_{h}\right), \nabla\left(v-v^{I}\right)\right)= & \sum_{K \in \mathcal{T}_{h}}\left(\nabla \cdot F\left(x, \nabla u_{h}\right), v-v^{I}\right)_{K} \\
& -\sum_{K \in \mathcal{T}_{h}}\left(F\left(x, \nabla u_{h}\right) \cdot \mathbf{n}_{K}, v-v^{I}\right)_{\partial K} .
\end{aligned}
$$

Then, $\Sigma_{1}$ in $(22)$ becomes

$$
\begin{aligned}
\Sigma_{1}= & \sum_{K \in \mathcal{T}_{h}}\left(\nabla \cdot F\left(x, \nabla u_{h}\right)-g\left(x, u_{h}, \nabla u_{h}\right), v-v^{I}\right)_{K} \\
& -\sum_{K \in \mathcal{T}_{h}}\left(F\left(x, \nabla u_{h}\right) \cdot \mathbf{n}_{K}, v-v^{I}\right)_{\partial K} .
\end{aligned}
$$

In view of the definition of $a(\cdot, \cdot)$, we have

$$
\begin{aligned}
a\left(u_{h}, v^{I}\right) & =\sum_{K \in \mathcal{T}_{h}}\left(F\left(x, \nabla u_{h}\right), \nabla v^{I}\right)_{K}+\sum_{K \in \mathcal{T}_{h}}\left(g\left(x, u_{h}, \nabla u_{h}\right), v^{I}\right) \\
& =I_{1}+I_{2} .
\end{aligned}
$$


Since $I_{h}^{*} v^{I}$ is a constant in $K \cap K_{p}^{*}, p \in N_{h}(K)$, from Green's formula, we have

$$
\begin{aligned}
I_{1}= & \sum_{K \in \mathcal{T}_{h}} \sum_{p \in N_{h}(K)} \int_{K \cap K_{p}^{*}} F\left(x, \nabla u_{h}\right) \cdot \nabla\left(v^{I}-I_{h}^{*} v^{I}\right) \mathrm{d} x \\
= & -\sum_{K \in \mathcal{T}_{h}} \sum_{p \in N_{h}(K)} \int_{K \cap K_{p}^{*}} \nabla \cdot F\left(x, \nabla u_{h}\right)\left(v^{I}-I_{h}^{*} v^{I}\right) \mathrm{d} x \\
& +\sum_{K \in \mathcal{T}_{h}} \sum_{p \in N_{h}(K)} \int_{\partial\left(K \cap K_{p}^{*}\right)} F\left(x, \nabla u_{h}\right) \cdot \mathbf{n}\left(v^{I}-I_{h}^{*} v^{I}\right) \mathrm{d} s \\
= & -\sum_{K \in \mathcal{T}_{h}} \sum_{p \in N_{h}(K)} \int_{K \cap K_{p}^{*}} \nabla \cdot F\left(x, \nabla u_{h}\right)\left(v^{I}-I_{h}^{*} v^{I}\right) \mathrm{d} x \\
& +\sum_{K \in \mathcal{T}_{h}} \int_{\partial K} F\left(x, \nabla u_{h}\right) \cdot \mathbf{n}\left(v^{I}-I_{h}^{*} v^{I}\right) \mathrm{d} s \\
& +\sum_{K \in \mathcal{T}_{h}} \sum_{p \in N_{h}(K)} \int_{K \cap \partial K_{p}^{*}} F\left(x, \nabla u_{h}\right) \cdot \mathbf{n}\left(v^{I}-I_{h}^{*} v^{I}\right) \mathrm{d} s .
\end{aligned}
$$

Since $F\left(x, \nabla u_{h}\right)$ and $v^{I}$ are continuous inside each $K \in \mathcal{T}_{h}$, we obtain

$$
\sum_{p \in N_{h}(K)} \int_{K \cap \partial K_{p}^{*}} F\left(x, \nabla u_{h}\right) \cdot \mathbf{n} v^{I} \mathrm{~d} s=0 .
$$

Noting that $\left.v^{I}\right|_{E}=\left.I_{h}^{*} v^{I}\right|_{E}=0, E \in \partial \Omega$, from (26) and (27), we have

$$
\begin{aligned}
I_{1}= & -\sum_{K \in \mathcal{T}_{h}}\left(\nabla \cdot F\left(x, \nabla u_{h}\right), v^{I}-I_{h}^{*} v^{I}\right)_{K}+\sum_{K \in \mathcal{T}_{h}}\left(F\left(x, \nabla u_{h}\right) \cdot \mathbf{n}, v^{I}-I_{h}^{*} v^{I}\right)_{\partial K} \\
& -\sum_{p \in N_{h}^{0}} v^{I}(p) \int_{\partial K_{p}^{*}} F\left(x, \nabla u_{h}\right) \cdot \mathbf{n} \mathrm{d} s .
\end{aligned}
$$

From (9), we know $a_{h}\left(u_{h}, I_{h}^{*} v^{I}\right)=0$, i.e.,

$$
-\sum_{p \in N_{h}^{0}} v^{I}(p) \int_{\partial K_{p}^{*}} F\left(x, \nabla u_{h}\right) \cdot \mathbf{n} \mathrm{d} s+\left(g\left(x, u_{h}, \nabla u_{h}\right), I_{h}^{*} v^{I}\right)=0 .
$$

Combining (25), (28) with (29) yields

$$
\begin{aligned}
\Sigma_{2}= & -a\left(u_{h}, v^{I}\right) \\
= & \sum_{K \in \mathcal{T}_{h}}\left(\nabla \cdot F\left(x, \nabla u_{h}\right)-g\left(x, u_{h}, \nabla u_{h}\right), v^{I}-I_{h}^{*} v^{I}\right)_{K} \\
& -\sum_{K \in \mathcal{T}_{h}}\left(F\left(x, \nabla u_{h}\right) \cdot \mathbf{n}, v^{I}-I_{h}^{*} v^{I}\right)_{\partial K} .
\end{aligned}
$$

Using (22), (24) and (30), we get

$$
\begin{aligned}
a(u, v)-a\left(u_{h}, v\right)= & \sum_{K \in \mathcal{T}_{h}}\left(\nabla \cdot F\left(x, \nabla u_{h}\right)-g\left(x, u_{h}, \nabla u_{h}\right), v-I_{h}^{*} v^{I}\right)_{K} \\
& -\sum_{K \in \mathcal{T}_{h}}\left(F\left(x, \nabla u_{h}\right) \cdot \mathbf{n}, v-I_{h}^{*} v^{I}\right)_{\partial K} .
\end{aligned}
$$


Since $v-I_{h}^{*} v^{I}$ is continuous on the interior edges and zero on the boundary edges, we have

$$
\sum_{K \in \mathcal{T}_{h}}\left(F\left(x, \nabla u_{h}\right) \cdot \mathbf{n}, v-I_{h}^{*} v^{I}\right)_{\partial K}=\sum_{E \in \mathcal{E}_{h}^{0}}\left(\left[F\left(x, \nabla u_{h}\right)\right], v-I_{h}^{*} v^{I}\right)_{E} .
$$

Then, the result of this lemma follows from (21), (31) and (32).

Motivated by the above lemma, we introduce locally computable quantities that play a major role in the residual-based a posteriori error analysis.

Definition 3.1. On each $K \in \mathcal{T}_{h}$ and $E \in \mathcal{E}_{h}^{0}$, define the residual $R_{K}$ and the jump $J_{E}$, respectively,

$$
\begin{gathered}
R_{K}=R_{K}\left(u_{h}\right)=\nabla \cdot F\left(x, \nabla u_{h}\right)-g\left(x, u_{h}, \nabla u_{h}\right), \\
J_{E}=J_{E}\left(u_{h}\right)=\left[F\left(x, \nabla u_{h}\right)\right]_{E}
\end{gathered}
$$

and define the local error estimators

$$
\eta_{R, K}^{2}=h_{K}^{2}\left\|R_{K}\right\|_{0, K}^{2}, \quad \eta_{J, E}^{2}=h_{E}\left\|J_{E}\right\|_{0, E}^{2}, \quad \eta_{E}^{2}=\sum_{K \in \omega_{E}} \eta_{R, K}^{2}+\eta_{J, E}^{2},
$$

where $\omega_{E}$ is the union of two elements which share the edge $E$.

The residual-based $H^{1}$-norm a posteriori error estimator is defined by

$$
\eta=\eta_{R}+\eta_{J}
$$

where

$$
\eta_{R}=\left(\sum_{K \in \mathcal{T}_{h}} \eta_{R, K}^{2}\right)^{\frac{1}{2}}, \quad \eta_{J}=\left(\sum_{E \in \mathcal{E}_{h}^{0}} \eta_{J, E}^{2}\right)^{\frac{1}{2}}
$$

We are now in a position to prove the main result in this subsection. It establishes a reliable estimate for the error $u-u_{h}$ in the $H^{1}$-norm.

Theorem 3.4. Assume that $u \in W^{2, r}(\Omega), r>2$, and $u_{h}$ are the solutions of (1) and (9), respectively. Then, there exists a constant $h_{1}>0$ such that $0<h \leq h_{1}$,

$$
\left\|u-u_{h}\right\|_{1} \leq C \eta
$$

Proof. In the proof, we will estimate the terms on the right-hand side of $(20)$ separately. It follows from Cauchy-Schwarz inequality, the interpolation error estimates and Lemma 2.1 that

$$
\begin{aligned}
\left|\Pi_{1}\right| & =\left|\sum_{K \in \mathcal{T}_{h}}\left(R_{K}, v-I_{h}^{*} v^{I}\right)_{K}\right| \\
& \leq\left(\sum_{K \in \mathcal{T}_{h}} h_{K}^{2}\left\|R_{K}\right\|_{0, K}^{2}\right)^{\frac{1}{2}}\left(\sum_{K \in \mathcal{T}_{h}} h_{K}^{-2}\left\|v-I_{h}^{*} v^{I}\right\|_{0, K}^{2}\right)^{\frac{1}{2}} \\
& \leq C \eta_{R}\left(\sum_{K \in \mathcal{T}_{h}} h_{K}^{-2}\left(\left\|v-v^{I}\right\|_{0, K}^{2}+\left\|v^{I}-I_{h}^{*} v^{I}\right\|_{0, K}^{2}\right)\right)^{\frac{1}{2}} \\
& \leq C \eta_{R}\|v\|_{1} .
\end{aligned}
$$


The estimation of $\Pi_{2}$ is obtained using Cauchy-Schwarz inequality, Lemma 2.6, Lemma 2.1 and the interpolation error estimates in the following calculation

$$
\begin{aligned}
\left|\Pi_{2}\right| & \leq \sum_{E \in \mathcal{E}_{h}^{0}}\left\|\left[F\left(x, \nabla u_{h}\right)\right]\right\|_{0, E} \cdot\left\|v-I_{h}^{*} v^{I}\right\|_{0, E} \\
& \leq\left(\sum_{E \in \mathcal{E}_{h}^{0}} h_{E}\left\|J_{E}\right\|_{0, E}^{2}\right)^{\frac{1}{2}} \cdot\left(\sum_{E \in \mathcal{E}_{h}^{0}} h_{E}^{-1}\left\|v-I_{h}^{*} v^{I}\right\|_{0, E}^{2}\right)^{\frac{1}{2}} \\
& \leq C \eta_{J}\left(\sum_{K \in \mathcal{T}_{h}}\left(h_{K}^{-2}\left\|v-I_{h}^{*} v^{I}\right\|_{0, K}^{2}+\left|v-I_{h}^{*} v^{I}\right|_{1, K}^{2}\right)\right)^{\frac{1}{2}} \\
& \leq C \eta_{J}\left(\sum_{K \in \mathcal{T}_{h}}\left(h_{K}^{-2}\left(\left\|v-v^{I}\right\|_{0, K}^{2}+\left\|v^{I}-I_{h}^{*} v^{I}\right\|_{0, K}^{2}\right)+|v|_{1, K}^{2}\right)\right)^{\frac{1}{2}} \\
& \leq C \eta_{J}\|v\|_{1} .
\end{aligned}
$$

Since $u \in W^{2, r}(\Omega), r>2$, using the Sobolev imbedding theorem [9] and Lemma 2.4 , we know that there exists a positive constant $K_{1}$ such that

$$
\|u\|_{1, \infty}+\left\|u_{h}\right\|_{1, \infty} \leq C\|u\|_{2,2+\epsilon} \leq K_{1} .
$$

Then, it follows from (19) and Lemma 2.5 that

$$
\begin{aligned}
\left|\Pi_{3}\right| & \leq C\left(\left\|e_{h}\right\|_{0,4}^{2}+\left\|\nabla e_{h}\right\|_{0,4}^{2}+\left\|e_{h} \nabla e_{h}\right\|\right)\|\nabla v\| \\
& \leq C\left(\left\|e_{h}\right\|_{0, \infty}\left\|e_{h}\right\|+\left\|\nabla e_{h}\right\|_{0, \infty}\left\|\nabla e_{h}\right\|+\left\|\nabla e_{h}\right\|_{0, \infty}\left\|e_{h}\right\|\right)\|\nabla v\| \\
& \leq C\left\|u-u_{h}\right\|_{1, \infty}\left\|e_{h}\right\|_{1}\|v\|_{1} \\
& \leq C_{1} h^{1-\frac{2}{r}}|\ln h||u|_{2, r}\left\|e_{h}\right\|\left\|_{1}\right\| v \|_{1} .
\end{aligned}
$$

From (20), (36)-(38), we get

$$
\frac{a^{\prime}\left(u, e_{h}, v\right)}{\|v\|_{1}} \leq C\left(\eta_{R}+\eta_{J}\right)+C_{1} h^{1-\frac{2}{r}}|\ln h||u|_{2, r}\left\|e_{h}\right\|_{1} .
$$

It follows from Lemma 3.2 that

$$
\left\|e_{h}\right\|_{1} \leq C\left(\eta_{R}+\eta_{J}\right)+C_{1} h^{1-\frac{2}{r}}|\ln h||u|_{2, r}\left\|e_{h}\right\|_{1} .
$$

There exists a constant $h_{1}>0$ such that for $0<h \leq h_{1}$,

$$
C_{1} h^{1-\frac{2}{r}}|\ln h||u|_{2, r} \leq \frac{1}{2}
$$

Therefore, the proof completes from (39) and (40).

3.2. Local lower bound. In this subsection, we derive the local lower bounds on the error $u-u_{h}$ in the $H^{1}$-norm. For this purpose, we shall use some properties of the bubble functions. For each triangle $K \in \mathcal{T}_{h}$, denote by $\lambda_{K, 1}, \lambda_{K, 2}, \lambda_{K, 3}$ the barycentric co-ordinates. Define the triangle-bubble function $b_{K}$ by

$$
b_{K}=\left\{\begin{array}{cc}
27 \lambda_{K, 1} \lambda_{K, 2} \lambda_{K, 3}, & \text { on } K, \\
0, & \text { on } \Omega \backslash K .
\end{array}\right.
$$

Furthermore, given two triangles $K$ and $K^{\prime}$ (such that $\omega_{E}=K \cup K^{\prime}$ ) sharing an interior edge $E$, we set

$$
b_{E}=\left\{\begin{array}{c}
4 \lambda_{K, k} \lambda_{K, j}, \quad \text { on } K \\
4 \lambda_{K^{\prime}, l} \lambda_{K^{\prime}, m}, \quad \text { on } K^{\prime} \\
0, \text { on } \Omega \backslash \omega_{E}
\end{array}\right.
$$


$\lambda_{K, k}$ and $\lambda_{K, j}$ are the barycentric coordinates associated with $K$ with $\lambda_{K, k} \lambda_{K, j}=0$ on $\partial K \backslash E$. The functions $\lambda_{K^{\prime}, l}$ and $\lambda_{K^{\prime}, m}$ are such that $\lambda_{K^{\prime}, l} \lambda_{K^{\prime}, n}=0$ on $\partial K^{\prime} \backslash E$.

Some properties of the bubble functions are collected in the following lemma.

Lemma 3.5. ([34]) For each element $K \in \mathcal{T}_{h}$ and each edge $E \in \mathcal{E}_{h}^{0}$, the functions $b_{K}$ and $b_{E}$ satisfy the following properties:

(1) $\operatorname{supp} b_{K} \subset K, 0 \leq b_{K} \leq 1$, and $\max _{x \in K} b_{K}(x)=1$;

$$
\int_{K} b_{K} \mathrm{~d} x=\frac{9}{20}|K| \sim h_{K}^{2}, \quad\left\|\nabla b_{K}\right\|_{0, K} \leq C h_{K}^{-1}\left\|b_{K}\right\|_{0, K} ;
$$

(2) $\operatorname{supp}_{E} \subset \omega_{E}, 0 \leq b_{E} \leq 1$, and $\max _{x \in \omega_{E}} b_{E}(x)=1 ; \int_{E} b_{E} \mathrm{ds}=\frac{2}{3} h_{E}$,

$$
\int_{\omega_{E}} b_{E} \mathrm{~d} x=\frac{1}{3}\left|\omega_{E}\right| \sim h_{E}^{2} ; \quad\left\|\nabla b_{E}\right\|_{0, \omega_{E}} \leq\left. C h_{E}^{-1}|| b_{E}\right|_{0, \omega_{E}} .
$$

The following integral form of the Taylor's formula for $\mathbf{p} \in \mathbb{R}^{2}$ in terms of $\mathbf{q} \in \mathbb{R}^{2}$ is used in the subsequent analysis

$$
F(x, \mathbf{p})-F(x, \mathbf{q})=-\widetilde{F}_{z}(x, \mathbf{q})(\mathbf{q}-\mathbf{p}),
$$

where

$$
\widetilde{F}_{z}(x, \mathbf{q})=\int_{0}^{1} F_{z}\left(x, \mathbf{p}^{t}\right) \mathrm{d} t, \quad \mathbf{p}^{t}=\mathbf{q}+t(\mathbf{p}-\mathbf{q}) .
$$

Similarly, the above formula can be defined for the function $g$ as

$$
g(v, \mathbf{p})-g(u, \mathbf{q})=-\widetilde{g}_{y}(u, \mathbf{q})(u-v)-\widetilde{g}_{z}(u, \mathbf{q})(\mathbf{q}-\mathbf{p}),
$$

where

$$
\begin{gathered}
\widetilde{g}_{y}(u, \mathbf{q})=\int_{0}^{1} g_{y}\left(v^{t}, \mathbf{p}^{t}\right) \mathrm{d} t, \\
\widetilde{g}_{z}(u, \mathbf{q})=\int_{0}^{1} g_{z}\left(v^{t}, \mathbf{p}^{t}\right) \mathrm{d} t, v^{t}=u+t(v-u) .
\end{gathered}
$$

To present the local lower bounds on the error $u-u_{h}$ in the $H^{1}$-norm, we introduce the oscillations of the residual $R_{K}$ and the jump $J_{E}$ :

$$
\operatorname{osc}_{R, K}=h_{K}\left\|R_{K}-\bar{R}_{K}\right\|_{0, K}, \quad \operatorname{osc}_{J, E}=h_{E}^{\frac{1}{2}}\left\|J_{E}-\bar{J}_{E}\right\|_{0, E},
$$

where $\bar{R}_{K}$ is the average of $R_{K}$ over $K$ and $\bar{J}_{E}$ is the average of $J_{E}$ on $E$, which are defined by, respectively,

$$
\bar{R}_{K}=\frac{1}{|K|} \int_{K} R_{K}\left(u_{h}\right) \mathrm{d} x, \quad \bar{J}_{E}=\frac{1}{h_{E}} \int_{E} J_{E}\left(u_{h}\right) \mathrm{d} s .
$$

We denote the total oscillations $\operatorname{osc}_{\mathcal{E}_{h}^{0}}$ by

$$
\operatorname{osc}_{\mathcal{E}_{h}^{0}}^{2}=\sum_{E \in \mathcal{E}_{h}^{0}} \operatorname{osc}_{E}^{2}, \quad \text { where } \quad \operatorname{osc}_{E}^{2}=\sum_{K \in \omega_{E}} \operatorname{osc}_{R, K}^{2}+\operatorname{osc}_{J, E}^{2} .
$$

Theorem 3.6. Assume that $u$ and $u_{h}$ are the solutions of (1) and (9), respectively. There exists a constant $C$ independent of $h_{K}$ and $h_{E}$ such that

(i). for each $K \in \mathcal{T}_{h}$,

$$
h_{K}\left\|R_{K}\right\|_{0, K} \leq C\left\|u-u_{h}\right\|_{1, K}+\operatorname{Cosc}_{R, K} .
$$


(ii). for each $E \in \mathcal{E}_{h}^{0}$,

$$
h_{E}^{\frac{1}{2}}\left\|J_{E}\right\|_{0, E} \leq C\left\|u-u_{h}\right\|_{1, \omega_{E}}+C \operatorname{osc}_{J, E}+C \sum_{K \in \omega_{E}} \operatorname{osc}_{R, K} .
$$

Proof. Assertion (i): It follows from triangle inequality that

$$
\begin{aligned}
h_{K}\left\|R_{K}\right\|_{0, K} & \leq h_{K}\left\|\bar{R}_{K}\right\|_{0, K}+h_{K}\left\|R_{K}-\bar{R}_{K}\right\|_{0, K} \\
& =h_{K}\left\|\bar{R}_{K}\right\|_{0, K}+\operatorname{osc}_{R, K} .
\end{aligned}
$$

Next, we only estimate $h_{K}\left\|\bar{R}_{K}\right\|_{0, K}$. By the properties of $b_{K}$ in Lemma 3.5

$$
\begin{aligned}
\frac{9}{20}\left\|\bar{R}_{K}\right\|_{0, K}^{2} & =\left(\bar{R}_{K}, b_{K} \bar{R}_{K}\right)_{K} \\
& =\left(R_{K}, b_{K} \bar{R}_{K}\right)_{K}+\left(\bar{R}_{K}-R_{K}, b_{K} \bar{R}_{K}\right)_{K} .
\end{aligned}
$$

Using Cauchy-Schwarz inequality and $\max _{x \in K} b_{K}(x)=1$, we get

$$
\begin{aligned}
\left|\left(\bar{R}_{K}-R_{K}, b_{K} \bar{R}_{K}\right)_{K}\right| & \leq\left\|R_{K}-\bar{R}_{K}\right\|_{0, K}\left\|b_{K} \bar{R}_{K}\right\|_{0, K} \\
& \leq\left\|R_{K}-\bar{R}_{K}\right\|_{0, K}\left\|\bar{R}_{K}\right\|_{0, K} .
\end{aligned}
$$

Since $b_{K} \bar{R}_{K} \in H_{0}^{1}(K) \subset H_{0}^{1}(\Omega)$, from $(14)$, we have

$$
a\left(u, b_{K} \bar{R}_{K}\right)=0 \text {, }
$$

$$
\left(F(x, \nabla u), \nabla\left(b_{K} \bar{R}_{K}\right)\right)_{K}+\left(g(x, u, \nabla u), b_{K} \bar{R}_{K}\right)_{K}=0 .
$$

By the definition of $R_{K}$, Green's formula and (51),

$$
\begin{aligned}
\left(R_{K}, b_{K} \bar{R}_{K}\right)_{K}= & \left(\nabla \cdot F\left(x, \nabla u_{h}\right), b_{K} \bar{R}_{K}\right)_{K}-\left(g\left(x, u_{h}, \nabla u_{h}\right), b_{K} \bar{R}_{K}\right)_{K} \\
= & -\left(F\left(x, \nabla u_{h}\right), \nabla\left(b_{K} \bar{R}_{K}\right)\right)_{K}-\left(g\left(x, u_{h}, \nabla u_{h}\right), b_{K} \bar{R}_{K}\right)_{K} \\
= & \left(F(x, \nabla u)-F\left(x, \nabla u_{h}\right), \nabla\left(b_{K} \bar{R}_{K}\right)\right)_{K} \\
& +\left(g(x, u, \nabla u)-g\left(x, u_{h}, \nabla u_{h}\right), b_{K} \bar{R}_{K}\right)_{K} \\
= & Q_{1}+Q_{2} .
\end{aligned}
$$

It follows from (41) and (43) that

$$
\begin{aligned}
F(x, \nabla u)-F\left(x, \nabla u_{h}\right)= & \widetilde{F}_{z}(x, \nabla u) \nabla\left(u-u_{h}\right), \\
g(x, u, \nabla u)-g\left(x, u_{h}, \nabla u_{h}\right)= & \widetilde{g}_{y}(x, u, \nabla u)\left(u-u_{h}\right) \\
& +\widetilde{g}_{z}(x, u, \nabla u) \nabla\left(u-u_{h}\right),
\end{aligned}
$$

where $\widetilde{F}_{z}, \widetilde{g}_{y}$ and $\widetilde{g}_{z}$ can be defined by (42), (44) and (45) similarly.

From (4), (53) and (54), Cauchy-Schwarz inequality and Lemma 3.5, we get

$$
\begin{aligned}
\left|Q_{1}\right| & \leq\left. C\left|u-u_{h}\right|_{1, K}|| \nabla\left(b_{K} \bar{R}_{K}\right)\right|_{0, K}=\left.C\left|u-u_{h}\right|_{1, K}|| \nabla b_{K}\right|_{0, K}\left|\bar{R}_{K}\right| \\
& \leq\left. C h_{K}^{-1}\left|u-u_{h}\right|_{1, K}|| b_{K}\right|_{0, K}\left|\bar{R}_{K}\right|=\left.C h_{K}^{-1}\left|u-u_{h}\right|_{1, K}|| b_{K} \bar{R}_{K}\right|_{0, K} \\
& \leq\left. C h_{K}^{-1}\left|u-u_{h}\right|_{1, K}|| \bar{R}_{K}\right|_{0, K},
\end{aligned}
$$

and

(56) $\quad\left|Q_{2}\right| \leq C|| u-u_{h}\left\|_{1, K}|| b_{K} \bar{R}_{K}\right\|_{0, K} \leq C|| u-u_{h}\left\|_{1, K}\right\| \bar{R}_{K} \|_{0, K}$.

From (48), (49), (52), (55), (56), we have

$$
\left\|\bar{R}_{K}\right\|_{0, K} \leq C h_{K}^{-1}\left\|u-u_{h}\right\|_{1, K}+C\left\|R_{K}-\bar{R}_{K}\right\|_{0, K},
$$

$$
h_{K}\left\|\bar{R}_{K}\right\|_{0, K} \leq C\left\|u-u_{h}\right\|_{1, K}+C \operatorname{Cosc}_{R, K} .
$$


Then, assertion (i) follows from (47) and (57).

Assertion (ii): By the triangle inequality once more,

$$
\begin{aligned}
h_{E}^{\frac{1}{2}}\left\|J_{E}\right\|_{0, E} & \leq h_{E}^{\frac{1}{2}}\left\|\bar{J}_{E}\right\|_{0, E}+h_{E}^{\frac{1}{2}}\left\|J_{E}-\bar{J}_{E}\right\|_{0, E} \\
& =h_{E}^{\frac{1}{2}}\left\|\bar{J}_{E}\right\|_{0, E}+\operatorname{osc}_{J, E} .
\end{aligned}
$$

Next, we only estimate $h_{E}^{\frac{1}{2}}\left\|\bar{J}_{E}\right\|_{0, E}$. By the properties of $b_{E}$ in Lemma 3.5

$$
\begin{aligned}
\frac{2}{3}\left\|\bar{J}_{E}\right\|_{0, E}^{2} & =\left(\bar{J}_{E}, b_{E} \bar{J}_{E}\right)_{E} \\
& =\left(J_{E}, b_{E} \bar{J}_{E}\right)_{E}+\left(\bar{J}_{E}-J_{E}, b_{E} \bar{J}_{E}\right)_{E} .
\end{aligned}
$$

Using Cauchy-Schwarz inequality and $\max _{x \in \omega_{E}} b_{E}(x)=1$ to get

$$
\begin{aligned}
\left|\left(\bar{J}_{E}-J_{E}, b_{E} \bar{J}_{E}\right)_{E}\right| & \leq\left\|\bar{J}_{E}-J_{E}\right\|_{0, E}\left\|b_{E} \bar{J}_{E}\right\|_{0, E} \\
& \leq\left\|\bar{J}_{E}-J_{E}\right\|_{0, E}\left\|\bar{J}_{E}\right\|_{0, E} .
\end{aligned}
$$

Since $b_{E} \bar{J}_{E} \in H_{0}^{1}\left(\omega_{E}\right) \subset H_{0}^{1}(\Omega)$, as in (51), we have

$$
\left(F(x, \nabla u), \nabla\left(b_{E} \bar{J}_{E}\right)\right)_{\omega_{E}}+\left(g(x, u, \nabla u), b_{E} \bar{J}_{E}\right)_{\omega_{E}}=0 .
$$

Since $b_{E} \bar{J}_{E} \in H_{0}^{1}\left(\omega_{E}\right)$, by the definition of $J_{E}$, Green's formula and (61),

$$
\begin{aligned}
\left(J_{E}, b_{E} \bar{J}_{E}\right)_{E}= & \left(\left[F\left(x, \nabla u_{h}\right)\right], b_{E} \bar{J}_{E}\right)_{E} \\
= & \left(F\left(x, \nabla u_{h}\right), \nabla\left(b_{E} \bar{J}_{E}\right)\right)_{\omega_{E}}+\left(\nabla_{h} \cdot F\left(x, \nabla u_{h}\right), b_{E} \bar{J}_{E}\right)_{\omega_{E}} \\
= & \left(F\left(x, \nabla u_{h}\right), \nabla\left(b_{E} \bar{J}_{E}\right)\right)_{\omega_{E}}+\left(g\left(x, u_{h}, \nabla u_{h}\right), b_{E} \bar{J}_{E}\right)_{\omega_{E}} \\
& +\left(\nabla_{h} \cdot F\left(x, \nabla u_{h}\right)-g\left(x, u_{h}, \nabla u_{h}\right), b_{E} \bar{J}_{E}\right)_{\omega_{E}} \\
= & \left(F\left(x, \nabla u_{h}\right)-F(x, \nabla u), \nabla\left(b_{E} \bar{J}_{E}\right)\right)_{\omega_{E}} \\
& +\left(g\left(x, u_{h}, \nabla u_{h}\right)-g(x, u, \nabla u), b_{E} \bar{J}_{E}\right)_{\omega_{E}} \\
& +\left(\nabla_{h} \cdot F\left(x, \nabla u_{h}\right)-g\left(x, u_{h}, \nabla u_{h}\right), b_{E} \bar{J}_{E}\right)_{\omega_{E}} \\
= & R_{1}+R_{2}+R_{3},
\end{aligned}
$$

where $\nabla_{h} \cdot F\left(x, \nabla u_{h}\right)$ is the function whose restriction to $K$ is $\nabla \cdot F\left(x, \nabla u_{h}\right)$. Since $\max _{x \in \omega_{E}} b_{E}(x)=1$, we get the estimation of $\left\|b_{E} \bar{J}_{E}\right\|_{0, \omega_{E}}$,

$$
\left\|\left.b_{E} \bar{J}_{E}\right|_{0, \omega_{E}}=\left.|| b_{E}\right|_{0, \omega_{E}}\left|\bar{J}_{E}\right| \leq C h_{E}\left|\bar{J}_{E}\right| \leq C h_{E}^{\frac{1}{2}}|| \bar{J}_{E}\right\|_{0, E} .
$$

Using (53), (4), Cauchy-Schwarz inequality, Lemma 3.5 and (63), we obtain

$$
\begin{aligned}
\left|R_{1}\right| & \leq C\left|u-u_{h}\right|_{1, \omega_{E}}|| \nabla\left(b_{E} \bar{J}_{E}\right)||_{0, \omega_{E}}=C\left|u-u_{h}\right|_{1, \omega_{E}}|| \nabla b_{E}||_{0, \omega_{E}}\left|\bar{J}_{E}\right| \\
& \leq\left. C h_{E}^{-1}\left|u-u_{h}\right|_{1, \omega_{E}}|| b_{E}\right|_{0, \omega_{E}}\left|\bar{J}_{E}\right|=C h_{E}^{-1}\left|u-u_{h}\right|_{1, \omega_{E}}|| b_{E} \bar{J}_{E}||_{0, \omega_{E}} \\
& \leq\left. C h_{E}^{-\frac{1}{2}}\left|u-u_{h}\right|_{1, \omega_{E}}|| \bar{J}_{E}\right|_{0, E} .
\end{aligned}
$$

From (54), (4), Cauchy-Schwarz inequality and (63), we have

(65) $\left|R_{2}\right| \leq C|| u-u_{h}\left\|_{1, \omega_{E}}\right\| b_{E} \bar{J}_{E}\left\|_{0, \omega_{E}} \leq C h_{E}^{\frac{1}{2}}\right\| u-u_{h}\left\|_{1, \omega_{E}}\right\| \bar{J}_{E} \|_{0, E}$.

By Cauchy-Schwarz inequality and (63),

$$
\begin{aligned}
\left|R_{3}\right| & \leq\left\|\nabla_{h} \cdot F\left(x, \nabla u_{h}\right)-g\left(x, u_{h}, \nabla u_{h}\right)\right\|_{0, \omega_{E}}\left\|b_{E} \bar{J}_{E}\right\|_{0, \omega_{E}} \\
& \leq C h_{E}^{\frac{1}{2}}\left\|\nabla_{h} \cdot F\left(x, \nabla u_{h}\right)-g\left(x, u_{h}, \nabla u_{h}\right)\right\|_{0, \omega_{E}}\left\|\bar{J}_{E}\right\|_{0, E} .
\end{aligned}
$$

Combining (59), (60), (62), (64), (65) with (66) yields

$$
\begin{aligned}
\left\|\bar{J}_{E}\right\|_{0, E} \leq & C h_{E}^{-\frac{1}{2}}\left\|u-u_{h}\right\|_{1, \omega_{E}}+C h_{E}^{\frac{1}{2}}\left\|\nabla_{h} \cdot F\left(x, \nabla u_{h}\right)-g\left(x, u_{h}, \nabla u_{h}\right)\right\|_{0, \omega_{E}} \\
& +\left\|\bar{J}_{E}-J_{E}\right\|_{0, E} .
\end{aligned}
$$


Multiplying $h_{E}^{\frac{1}{2}}$ on the both sides of (67), and applying (58) and assertion (i), we can obtain assertion (ii).

Remark 3.1. From Lemma 2.3, we know that the finite volume element method is of first order in the $H^{1}$-norm, i.e., $\left\|u-u_{h}\right\|_{1} \leq C h|u|_{2}$. In contrast to this estimate, ${ }^{\circ C} \mathcal{E}_{h}^{0}$ is higher order term, which tends to zero faster than $\mathcal{O}(h)$, see [12] for details. Then, from Theorems 3.4 and 3.6, we see that $\eta$ is a reliable and efficient a posteriori error estimator of $\left\|u-u_{h}\right\|_{1}$.

\section{4. $L^{2}$-norm a posteriori error estimator: global upper bound}

In this section, we propose a residual-based $L^{2}$-norm a posteriori error estimator of the finite volume element method for (1) which satisfy $D_{z z} F=0$ and $D_{z z} g=0$, such as Bratu's equation and nonlinear eigenvalue equation. We use the duality argument to derive the global upper bound on the approximation error in the $L^{2}$ norm.

For this purpose, we introduce the following auxiliary problem: find $\omega \in H_{0}^{1}(\Omega)$ such that

$$
a^{\prime}(u ; v, \omega)=\left(u-u_{h}, v\right), \quad \forall v \in H_{0}^{1}(\Omega) .
$$

By [24], we have the following elliptic regularity estimate

$$
\|\omega\|_{2} \leq C\left\|u-u_{h}\right\|
$$

Definition 4.1. We define the residual-based $L^{2}$-norm a posteriori error estimator $\tilde{\eta}$ as

$$
\tilde{\eta}=\tilde{\eta}_{R}+\tilde{\eta}_{J}
$$

where

$$
\begin{gathered}
\tilde{\eta}_{R}=\left(\sum_{K \in \mathcal{T}_{h}}\left(h_{K}^{2}\left\|R_{K}-\bar{R}_{K}\right\|_{0, K}^{2}+h_{K}^{4}\left\|R_{K}\right\|_{0, K}^{2}\right)\right)^{\frac{1}{2}}, \\
\tilde{\eta}_{J}=\left(\sum_{E \in \mathcal{E}_{h}^{0}}\left(h_{E}\left\|J_{E}-\bar{J}_{E}\right\|_{0, E}^{2}+h_{E}^{3}\left\|J_{E}\right\|_{0, E}^{2}\right)\right)^{\frac{1}{2}} .
\end{gathered}
$$

The following Theorem 4.1 gives a reliable estimate for the error in the $L^{2}$-norm.

Theorem 4.1. Assume that the problems (1) satisfy $D_{z z} F=0$ and $D_{z z} g=0$, $u \in W^{2, r}(\Omega), r>2$, and $u_{h}$ are the solutions of (1) and (9), respectively. Then, there exists a constant $h_{2}>0$ such that $0<h \leq h_{2}$,

$$
\left\|u-u_{h}\right\| \leq C \tilde{\eta}
$$

Proof. Let $v=u-u_{h}=e_{h}$ in (68), from (20) and the definitions of $R_{K}$ and $J_{E}$, we have

$$
\begin{aligned}
\left\|u-u_{h}\right\|^{2} & =a^{\prime}\left(u ; e_{h}, \omega\right) \\
& =\sum_{K \in \mathcal{T}_{h}}\left(R_{K}, \omega-I_{h}^{*} \omega^{I}\right)_{K}-\sum_{E \in \mathcal{E}_{h}^{0}}\left(J_{E}, \omega-I_{h}^{*} \omega^{I}\right)_{E}+R\left(u, u_{h}, \omega\right) \\
& =T_{1}+T_{2}+T_{3} .
\end{aligned}
$$


It follows from Lemma 2.1 that

$$
\begin{aligned}
T_{1} & =\sum_{K \in \mathcal{T}_{h}}\left(R_{K}, \omega-\omega^{I}\right)_{K}+\sum_{K \in \mathcal{T}_{h}}\left(R_{K}, \omega^{I}-I_{h}^{*} \omega^{I}\right)_{K} \\
& =\sum_{K \in \mathcal{T}_{h}}\left(R_{K}, \omega-\omega^{I}\right)_{K}+\sum_{K \in \mathcal{T}_{h}}\left(R_{K}-\bar{R}_{K}, \omega^{I}-I_{h}^{*} \omega^{I}\right)_{K} \\
& =T_{11}+T_{12} .
\end{aligned}
$$

Using Cauchy-Schwarz inequality, the interpolation estimate and (69), we get

$$
\begin{aligned}
\left|T_{11}\right| & \leq\left(\sum_{K \in \mathcal{T} h} h_{K}^{4}\left\|R_{K}\right\|_{0, K}^{2}\right)^{\frac{1}{2}}\left(\sum_{K \in \mathcal{T} h} h_{K}^{-4}\left\|\omega-\omega^{I}\right\|_{0, K}^{2}\right)^{\frac{1}{2}} \\
& \leq C\left(\sum_{K \in \mathcal{T} h} h_{K}^{4}\left\|R_{K}\right\|_{0, K}^{2}\right)^{\frac{1}{2}}\|\omega\|_{2} \\
& \leq C\left(\sum_{K \in \mathcal{T} h} h_{K}^{4}\left\|R_{K}\right\|_{0, K}^{2}\right)^{\frac{1}{2}}\left\|u-u_{h}\right\| .
\end{aligned}
$$

Similarly, from Cauchy-Schwarz inequality, Lemma 2.1 and $\left\|\omega^{I}\right\|_{1} \leq C\|\omega\|_{2} \leq$ $C\left\|u-u_{h}\right\|$, we get

$$
\begin{aligned}
\left|T_{12}\right| & \leq\left(\sum_{K \in \mathcal{T} h} h_{K}^{2}\left\|R_{K}-\bar{R}_{K}\right\|_{0, K}^{2}\right)^{\frac{1}{2}}\left(\sum_{K \in \mathcal{T} h} h_{K}^{-2}\left\|\omega^{I}-I_{h}^{*} \omega^{I}\right\|_{0, K}^{2}\right)^{\frac{1}{2}} \\
& \leq C\left(\sum_{K \in \mathcal{T} h} h_{K}^{2}\left\|R_{K}-\bar{R}_{K}\right\|_{0, K}^{2}\right)^{\frac{1}{2}}\left\|\omega^{I}\right\|_{1} \\
& \leq C\left(\sum_{K \in \mathcal{T} h} h_{K}^{2}\left\|R_{K}-\bar{R}_{K}\right\|_{0, K}^{2}\right)^{\frac{1}{2}}\left\|u-u_{h}\right\| .
\end{aligned}
$$

Combining (74), (75) with (73) yields

$$
\left|T_{1}\right| \leq C \tilde{\eta}_{R}|| u-u_{h} \|
$$

Using the technique to estimate $T_{1}$, we can bound the second term on the right-hand side of (72) in which Lemma 2.6 is used

$$
\left|T_{2}\right| \leq C \tilde{\eta}_{J}\left\|u-u_{h}\right\|
$$

Since $D_{z z} F=0$ and $D_{z z} g=0$, from (16), we know that $\delta_{1}=0$. Then, the third term on the right-hand side of (72) is estimated by (19), Lemma 2.5 and $|\omega|_{1} \leq\|\omega\|_{2} \leq C\left\|u-u_{h}\right\|$

$$
\begin{aligned}
\left|T_{3}\right| & \leq C\left(\left\|u-u_{h}\right\|_{0,4}^{2}+\left\|\left(u-u_{h}\right) \nabla\left(u-u_{h}\right)\right\|\right)\|\nabla \omega\| \\
& \leq C\left(\left\|u-u_{h}\right\|_{0, \infty}\left\|u-u_{h}\right\|+\left\|\nabla\left(u-u_{h}\right)\right\|_{0, \infty}\left\|u-u_{h}\right\|\right)\|\nabla \omega\| \\
& \leq C\left\|u-u_{h}\right\|_{1, \infty} \| u-u_{h}|||\omega|_{1} \\
& \leq C_{2} h^{1-\frac{2}{r}}|\ln h||u|_{2, r}\left\|u-u_{h}\right\|^{2}
\end{aligned}
$$

There exists a constant $h_{2}>0$ such that for $0<h \leq h_{2}$,

$$
C_{2} h^{1-\frac{2}{r}}|\ln h||u|_{2, r} \leq \frac{1}{2}
$$


From (72), (76)-(79), we get

$$
\frac{1}{2}\left\|u-u_{h}\right\|^{2} \leq C\left(\tilde{\eta}_{R}+\tilde{\eta}_{J}\right)\left\|u-u_{h}\right\|
$$

which together with (70) completes the proof.

Remark 4.1. Since Bratu's equation satisfies $D_{z z} F=0$ and $D_{z z} g=0, \tilde{\eta}$ is a reliable a posteriori error estimator of $\left\|u-u_{h}\right\|$. In this case, we have $J_{E}-\bar{J}_{E}=$ $0, \forall E \in \mathcal{E}_{h}^{0}$, and $\tilde{\eta}_{J}$ in $\tilde{\eta}$ is reduced to $\tilde{\eta}_{J}=\left(\sum_{E \in \mathcal{E}_{h}^{0}} h_{E}^{3}\left\|J_{E}\right\|_{0, E}^{2}\right)^{1 / 2}$.

\section{Numerical Experiments}

In this section, we present two numerical examples to illustrate the performance of the error estimators that have been analyzed earlier. Our particular interest is to give an observation about the ability of the a posteriori error estimator $\eta$ to imitate the convergence behavior of the exact errors in the $H^{1}$-norm for the nonlinear elliptic problems (1), as well as the residual-based $L^{2}$-norm a posteriori error estimator $\tilde{\eta}$ discussed in section 4.

In all examples, we use the same true solution

$$
u\left(x_{1}, x_{2}\right)=x_{1}^{3} \ln \left(x_{1}\right) x_{2}^{3} \ln \left(x_{2}\right),
$$

designate the expression of $F(x, u, \nabla u)$, and set the function $g(x, u)$ to satisfy the equation (1). For these two examples, the problems are posed in the domain $\Omega=$ $(0,1) \times(0,1)$. The domain is discretized into $N$ numbers of rectangle in each direction, and then each rectangle is divided into two triangles, resulting a mesh with size $h=\sqrt{2} / N$. For the computation, the conforming linear finite element space $S_{h}$ and the dual volume element space $S_{h}^{*}$ are built on these meshes and their dual partitions, respectively.

For the first example, we consider the equation of prescribed mean curvature described in subsection 2.1. The numerical results are shown in Table 1. Here and thereafter the comparisons of the error estimators $\eta$ and $\tilde{\eta}$ against the exact errors in the $H^{1}$ and $L^{2}$-norms are denoted by, respectively,

$$
R_{1}=\frac{\left\|u-u_{h}\right\|_{1}}{\eta}, \quad R_{2}=\frac{\left\|u-u_{h}\right\|}{\tilde{\eta}},
$$

where $\eta=\eta_{R}+\eta_{J}$ and $\tilde{\eta}=\tilde{\eta}_{R}+\tilde{\eta}_{J}$. From this table, we can see the same convergence rate of the a posterior error estimator $\eta$ and the exact error in the $H^{1}$-norm.

TABLE 1. Performance of the estimator $\eta$ for the first example.

\begin{tabular}{cccccc}
\hline$h$ & $\left\|u-u_{h}\right\|_{1}$ & $\eta_{R}$ & $\eta_{J}$ & $\eta$ & $R_{1}$ \\
\hline$\sqrt{2} / 10$ & $8.7736 \mathrm{e}-3$ & $2.0395 \mathrm{e}-2$ & $4.1867 \mathrm{e}-2$ & $6.2262 \mathrm{e}-2$ & 0.14 \\
\hline$\sqrt{2} / 20$ & $4.4137 \mathrm{e}-3$ & $1.0194 \mathrm{e}-2$ & $2.2572 \mathrm{e}-2$ & $3.2766 \mathrm{e}-2$ & 0.13 \\
\hline$\sqrt{2} / 40$ & $2.2110 \mathrm{e}-3$ & $5.0976 \mathrm{e}-3$ & $1.1653 \mathrm{e}-2$ & $1.6751 \mathrm{e}-2$ & 0.13 \\
\hline$\sqrt{2} / 80$ & $1.1078 \mathrm{e}-3$ & $2.5490 \mathrm{e}-3$ & $5.9128 \mathrm{e}-3$ & $8.4617 \mathrm{e}-3$ & 0.13 \\
\hline$\sqrt{2} / 160$ & $5.5776 \mathrm{e}-4$ & $1.2743 \mathrm{e}-3$ & $2.9772 \mathrm{e}-3$ & $4.2515 \mathrm{e}-3$ & 0.13 \\
\hline$\sqrt{2} / 320$ & $2.8635 \mathrm{e}-4$ & $6.3715 \mathrm{e}-4$ & $1.4937 \mathrm{e}-3$ & $2.1309 \mathrm{e}-3$ & 0.13 \\
\hline
\end{tabular}


For the second example, we consider the following semilinear problem.

$$
\left\{\begin{aligned}
-\triangle u+u^{3} & =f, & & \text { in } \quad \Omega, \\
u & =0, & & \text { on } \quad \partial \Omega,
\end{aligned}\right.
$$

where as before, $f(x)$ is chosen such that $(80)$ is the solution of the above problem. The numerical results are shown in Table 2 and 3 , which also confirm our theoretical results.

TABLE 2. Performance of the estimator $\eta$ for the second example.

\begin{tabular}{cccccc}
\hline$h$ & $\left\|u-u_{h}\right\|_{1}$ & $\eta_{R}$ & $\eta_{J}$ & $\eta$ & $R_{1}$ \\
\hline$\sqrt{2} / 10$ & $8.7712 \mathrm{e}-3$ & $2.4624 \mathrm{e}-3$ & $4.1914 \mathrm{e}-2$ & $4.4376 \mathrm{e}-2$ & 0.20 \\
\hline$\sqrt{2} / 20$ & $4.4120 \mathrm{e}-3$ & $1.2313 \mathrm{e}-3$ & $2.2605 \mathrm{e}-2$ & $2.3836 \mathrm{e}-2$ & 0.19 \\
\hline$\sqrt{2} / 40$ & $2.2092 \mathrm{e}-3$ & $6.1564 \mathrm{e}-4$ & $1.1672 \mathrm{e}-2$ & $1.2288 \mathrm{e}-2$ & 0.18 \\
\hline$\sqrt{2} / 80$ & $1.1050 \mathrm{e}-3$ & $3.0782 \mathrm{e}-4$ & $5.9229 \mathrm{e}-3$ & $6.2307 \mathrm{e}-3$ & 0.18 \\
\hline$\sqrt{2} / 160$ & $5.5255 \mathrm{e}-4$ & $1.5391 \mathrm{e}-4$ & $2.9825 \mathrm{e}-3$ & $3.1364 \mathrm{e}-3$ & 0.18 \\
\hline$\sqrt{2} / 320$ & $2.7628 \mathrm{e}-4$ & $7.6955 \mathrm{e}-5$ & $1.4964 \mathrm{e}-3$ & $1.5734 \mathrm{e}-3$ & 0.18 \\
\hline
\end{tabular}

TABLE 3. Performance of the estimator $\tilde{\eta}$ for the second example

\begin{tabular}{cccccc}
\hline$h$ & $\left\|u-u_{h}\right\|$ & $\tilde{\eta}_{R}$ & $\tilde{\eta}_{J}$ & $\tilde{\eta}$ & $R_{2}$ \\
\hline$\sqrt{2} / 10$ & $2.7726 \mathrm{e}-4$ & $3.7123 \mathrm{e}-4$ & $2.9638 \mathrm{e}-3$ & $3.3350 \mathrm{e}-3$ & 0.08 \\
\hline$\sqrt{2} / 20$ & $7.0263 \mathrm{e}-5$ & $9.3454 \mathrm{e}-5$ & $7.9920 \mathrm{e}-4$ & $8.9265 \mathrm{e}-4$ & 0.08 \\
\hline$\sqrt{2} / 40$ & $1.7631 \mathrm{e}-5$ & $2.3404 \mathrm{e}-5$ & $2.0633 \mathrm{e}-4$ & $2.2974 \mathrm{e}-4$ & 0.08 \\
\hline$\sqrt{2} / 80$ & $4.4021 \mathrm{e}-6$ & $5.8534 \mathrm{e}-6$ & $5.2351 \mathrm{e}-5$ & $5.8205 \mathrm{e}-5$ & 0.08 \\
\hline$\sqrt{2} / 160$ & $1.0888 \mathrm{e}-6$ & $1.4635 \mathrm{e}-6$ & $1.3181 \mathrm{e}-5$ & $1.4644 \mathrm{e}-5$ & 0.07 \\
\hline$\sqrt{2} / 320$ & $2.6008 \mathrm{e}-7$ & $3.6589 \mathrm{e}-7$ & $3.3066 \mathrm{e}-6$ & $3.6725 \mathrm{e}-6$ & 0.07 \\
\hline
\end{tabular}

\section{Summary and concluding remarks}

In this paper we established the a posteriori error estimates of the finite volume element method for the quasilinear elliptic problem (1). We derived the global upper and local lower bounds on the approximation error in the $H^{1}$-norm and developed the global upper bound on the error in the $L^{2}$-norm for some special quasilinear elliptic problems. Numerical experiments provided in this paper confirmed our theoretical findings.

The results of this paper can be extended to the following general nonlinear elliptic problems by making appropriate modification in the analysis

$$
\mathcal{L} u=-\nabla \cdot F(x, u, \nabla u)+g(x, u, \nabla u)=0, \quad \text { in } \quad \Omega, \quad u=0, \quad \text { on } \quad \partial \Omega .
$$

For the assumptions on the functions $F(x, y, z), g(x, y, z)$ and the operator $\mathcal{L}$, we refer to $[8,37]$ for details.

The a posteriori error estimator is an essential ingredient and also a major difficulty in the convergence analysis of an adaptive finite volume element method. 
Based on the error estimator described in this paper, we will develop and analyze an adaptive finite volume element method to solve (1) in the future work.

\section{References}

[1] D. N. Arnold, An interior penalty finite element method with discontinuous elements, SIAM J. Numer. Anal., 19 (1982) 742-760.

[2] I. Babuška and A. K. Aziz, Foundations of the finite element method, The Mathmetical Foundation of the Finite Element Method with Appications to Partial Differential Equations, A.K. Aziz, editor. New York: Academic Press, 1972, 3-362.

[3] R. E. Bank and D. J. Rose, Some error estimates for the box method. SIAM J. Numer. Anal., 24 (1987) $777-787$.

[4] A. Bergam, Z. Mghazli and R. Verfürth, Estimations a posteriori d'un schéma de volumes finis pour un problème non linéaire. Numer. Math., 95 (2003) 599-624.

[5] C. Bi, Superconvergence of finite volume element method for a nonlinear elliptic problem, Numer. Methods PDEs, 23 (2007) 220-233.

[6] C. Bi and V. Ginting, Two-grid finite volume element method for linear and nonlinear elliptic problems, Numer. Math., 108 (2007) 177-198.

[7] C. Bi and V. Ginting, A residual-type a posteriori error estimate of finite volume element method for a quasi-linear elliptic problem, Numer. Math., 114 (2009) 107-132.

[8] C. Bi and V. Ginting, Finite volume element method for second-order quasilinear elliptic problems, IMA J. Numer. Anal., 31 (2011) 1062-1089.

[9] S. C. Brenner and L. R. Scott, The mathematical theory of finite element methods, Texts in Applied Mathematics, vol.15, third edn. New York: Springer, 2008.

[10] Z. Cai, On the finite volume element method, Numer. Math., 58 (1991) 713-735.

[11] Z. Cai, J. Mandel and S. McCormick, The finite volume element method for diffusion equations on general triangulations, SIAM J. Numer. Anal., 28 (1991) 392-402.

[12] C. Carstensen, R. D. Lazarov and S. Tomov, Explicit and averaging a posteriori error estimates for adaptive finite volume methods, SIAM J. Numer. Anal., 42 (2005) 2496-2521.

[13] P. Chatzipantelidis, Finite volume methods for elliptic PDE's: a new approach, M2AN Math. Model. Numer. Anal., 36 (2002) 307-324.

[14] P. Chatzipantelidis, V. Ginting and R.D. Lazarov, A finite volume element method for a non-linear elliptic problem, Numer. Linear Algebra Appl., 12 (2005) 515-546.

[15] C. Chen and W. Liu, A two-grid finite volume element method for a nonlinear parabolic problem, Inter. J. Numer. Anal. Mod. 12 (2015) 197-210.

[16] S. H. Chou and D.Y. Kwak, Multigrid algorithms for a vertex-centered covolume method for elliptic problems, Numer. Math., 90 (2002) 441-458.

[17] S. H. Chou and Q. Li, Error estimates in $L^{2}-H^{1}$ - and $L^{\infty}$ - in covolume methods for elliptic and parabolic problems: a unified approach, Math. Comp., 69 (2000) 103-120.

[18] S. H. Chou and X. Ye, Unified analysis of finite volume methods for second order elliptic problems, SIAM J. Numer. Anal., 45 (2007) 1639-1653.

[19] P. G. Ciarlet, The Finite Element Method for Elliptic Problems, North-Holland, Amsterdam, 1978.

[20] A. Demlow, Localized pointwise a posteriori error estimates for gradients of piecewise linear finite element approximations to second-order quadilinear elliptic problems, SIAM J. Numer. Anal., 44 (2006) 494-514.

[21] W. Dörfler and M. Rumpf, An adaptive strategy for elliptic problems including a posteriori controlled boundary approximation, Math. Comp., 67 (1998) 1361-1382.

[22] R. E. Ewing, R. D. Lazarov and Y. Lin, Finite volume element approximations of nonlocal reactive flows in porous media, Numer. Methods PDEs, 16 (2000) 285-311.

[23] R. E. Ewing, T. Lin and Y. Lin, On the accuracy of the finite volume element method based on piecewise linear polynomials, SIAM J. Numer. Anal., 39 (2002) 1865-1888.

[24] P. Grisvard, Elliptic problems in nonsmooth domains, Monographs and Studies in Mathematics, vol. 24. Boston, MA: Pitman, 1985.

[25] T. Gudi, N. Nataraj and A. K. Pani, $h p$-discontinuous Galerkin methods for strongly nonlinear elliptic boundary value problems, Numer. Math., 109 (2008) 233-268.

[26] J. Huang and S. Xi, On the finite volume element method for general self-adjoint elliptic problems, SIAM J. Numer. Anal. 35 (1998) 1762-1774.

[27] S. Karaa and A. K. Pani, A priori error estimates for finite volume element approximations to second order linear hyperbolic integro-differential equations, Inter. J. Numer. Anal. Mod., 12 (2015) 401-429. 
[28] R. D. Lazarov and S. Tomov, A posteriori error estimates for finite volume element approximations of convection-diffusion-reaction equations, Comput. Geosci., 6 (2002) 483-503.

[29] R. Li, Generalized difference methods for a nonlinear Dirichlet problem. SIAM J. Numer. Anal., 24 (1987) 77-88.

[30] R. Li, Z. Chen and W. Wu, Generalized difference methods for differential equations, Monographs and Textbooks in Pure and Applied Mathematics, vol. 226. New York: Marcel Dekker Inc., 2000.

[31] X. Luo, Y. Chen and Y. Huang, Some error estimates of finite volume element approximation for elliptic optimal control problems, Inter. J. Numer. Anal. Mod., 10 (2013) 697-711.

[32] I. D. Mishev, Finite volume element methods for non-definite problems, Numer. Math., 83 (1999) 161-175.

[33] R. Verfürth, A posteriori error estimates for nonlinear problems. Finite element discretizations of elliptic equations, Math. Comp., 62 (1994) 445-475.

[34] R. Verfürth, A Review of A Posteriori Error Estimation and Adaptive Mesh-Refinement Techniques, Wiley-Teubner, Chichester, 1996.

[35] H. Wu and R. Li, Error estimates for finite volume element methods for general second-order elliptic problems, Numer. Methods PDEs, 19 (2003) 693-708.

[36] Z. Xiong and Y. Chen, A rectangular finite volume element method for a semilinear elliptic equation, J. Sci. Comput., 36 (2008) 177-191.

[37] J. Xu, Two-grid discretization techniques for linear and nonlinear PDEs, SIAM J. Numer. Anal., 33 (1996) 1759-1777.

[38] J. Xu and Q. Zou, Analysis of linear and quadratic simplicial finite volume methods for elliptic equations, Numer. Math., 111 (2009) 469-492.

[39] M. Yang and J. Liu, A quadratic finite volume element method for parabolic problems on quadrilateral meshes, IMA J Numer. Anal., 31 (2011) 1038-1061.

[40] M. Yang, J. Liu and Y. Lin, Quadratic finite volume methods for elliptic and parabolic problems on quadrilateral meshes: Optimal order errors based on barlow points, IMA J Numer. Anal., 33 (2013) 1042-1064.

[41] X. Ye, A new discontinuous finite volume method for elliptic problems, SIAM J. Numer. Anal., 42 (2004) 1062-1072.

[42] X. Ye, A discontinuous finite volume method for the Stokes problems, SIAM J. Numer. Anal., 44 (2006) 183-198.

Department of Mathematics, Yantai University, Shandong, 264005, People's Republic of China. E-mail: bicj@ytu.edu.cn

Department of Mathematics, Tongji University, Shanghai, 200092, People's Republic of China. E-mail: wangcheng@tongji.edu.cn 